Background Role of endoscopic ultrasound (EUS) guided fine needle aspiration (FNA) in patients with lymphadenopathy in term of diagnostic adequacy and safety in a large population is not well defined. The aim of the current study was to evaluate diagnostic adequacy and safety of EUS FNA in patients with lymphadenopathy.

Methods Retrospective study from October 2010 to September 2015 at a tertiary care centre in Delhi-NCR. We analysed data of 1005 EUS guided FNA of the lymph node.

Results The study cohort comprised 1005 lymph nodes in 865 patients; $68 \%$ were males, mean age was $50 \pm 14$ years. Indications of FNA were to look for aetiology of pyrexia of unknown origin or staging of malignancy mainly. lymph node FNA was taken from mediastinal nodes $(\mathrm{n}=528,52.5 \%)$ and intra-abdominal nodes $(n=477,47.5 \%)$. The median size of nodes at the long axis and short axis was 17 (12-25.7) and $10(8-15) \mathrm{mm}$ respectively. Adequate material by FNA was obtained in $92.8 \%$ cases. The cytopathologic diagnosis was malignancy in 153 (15.2\%), granulomatous change in 452 (42\%), and reactive lymphadenopathy in 328 (35.6\%). There was statistically significant difference seen between groups with pathological and reactive lymph nodes regarding size at the long and short axis, all the echo-features of lymph nodes. Procedure related adverse effects were encountered in 6 patients $(0.8 \%)$. Four patients had mild mucosal bleeding in chronic liver disease patients, and two had mild hepatic encephalopathy related to sedation.

Conclusions EUS FNA of lymph nodes has good diagnostic adequacy and safety.

\section{IDDF2018-ABS-0023 BUTYRATE ACID AS A POTENTIAL MARKER FOR THE DIVERSITY OF GUT MICROBIOTA IN COLORECTAL CANCER PATIENTS}

${ }^{1}$ Siti Adewiah*, ${ }^{2}$ Azzaki Abubakar, ${ }^{2}$ Fauzi Yusuf. ${ }^{1}$ Department of Internal Medicine, Faculty of Medicine, Syiah Kuala University, Banda Aceh, Indonesia; ${ }^{2}$ Division of GastroenterologyHepatology, Department of Internal Medicine, Faculty of Medicine University of Syiah Kuala/ Dr.Zainoel Abidin General Teaching Hospital Banda Aceh, Indonesia

\subsection{6/gutjnl-2018-IDDFabstracts.72}

Background The gut microbiota turns as an actual organism, and many changes of variety have been described in colorectal cancer patients (CRC). Butyrate is a part of short chain fatty acid which commonly produced by especially bacteria in the colon, and it was to use of their host. Several kinds of literature reported the role of butyrate as prevention or inhibition of carcinogenesis of intestinal. In addition, there is an opposite the link between butyrate acid's level and the rate of frequency CRC patients. In this study, we evaluated the level of butyrate acid as diagnostic biomarkers for the diversity of gut microbiota in colorectal cancer patients.

Methods The study consists of fourteen subjects with CRC and 14 non-CRC were included in this case-control study. Stool samples were analysed for SCFA (acetate, propionate, and butyrate acids) with gas chromatography and the result is given as $\mathrm{ug} / \mathrm{mL}$. Especially, we determined various cut-off marks for butyrate acids in respect of indicating CRC for addition to the computation of both sensitivity and specificity. Results We found that CRC patients had a lower level of acetate, propionate and butyrate acids than non-CRC. The mean concentration of acetate $8,55 \mathrm{ug} / \mathrm{mL}$, propionate $5,61 \mathrm{ug} / \mathrm{mL}$ and butyrate acids $3,79 \mathrm{ug} / \mathrm{mL}$ respectively. In three of SCFA, the level of butyrate acids had the best diagnostic properties with area under receiver operating characteristic (ROC) curve of 0.84 higher than acetate $(0.71)$ and propionate $(0.75)$ $(\mathrm{p}<0.05)$. With a cut-off value for butyrate acids $<5.4 \mathrm{ug} / \mathrm{mL}$ indicating CRC, the sensitivity, specificity, positive and negative likelihood ratio, and diagnostic odds ratio were $85 \%$, $78 \%, 4.04,0.18$ and 22.2 respectively.

Conclusions In conclusion, our study is the first report demonstrating the level butyrate acids as useful biomarkers to detect the presence of CRC. Due to the small size of the sample and this design study is case-control, needs more require approval to confirm the result.

\section{IDDF2018-ABS-0024 ORAL HEALTH STATUS OF ULCERATIVE COLITIS PATIENTS: A COMPARATIVE STUDY}

Delfin Lovelina Francis*. Tamil Nadu Dr MGR Medical University, India

\subsection{6/gutjnl-2018-IDDFabstracts.73}

Background Inflammatory bowel disease (IBD) is a complex civilization disease and has a prevalence of up to $0.2 \%$ in the Asian countries. The disease preferentially manifests in the second and third decade of life. Oral soft tissue lesions can precede or occur concomitantly with the intestinal symptoms. It was reported that IBD patients have a higher prevalence of dental caries than healthy controls as a result of nutritional deficiencies and changes in salivary and microbiological conditions in the oral cavity. Prior studies showed a higher prevalence of aphthous ulcer and dental caries. There is only a little information about the oral health status of UC patient, as often they are dealt only by the general physician. The aim of this study was to assess dental caries and periodontal disease status in patients with ulcerative colitis - an inflammatory bowel disease.

Methods After obtaining ethical clearance from the institutional review board of Lifeline hospital, a record based casecontrol study was conducted upon 20 UC patients and 20 healthy controls. Oral hygiene habits, Oral soft tissue changes, the decayed, missing and filled tooth surface (DMF - S) index, periodontal status (CPI) index and clinical attachment loss (CAL) were evaluated in each patient and in the controls. Results In patients with UC,28 oral lesions were found compared with 3 lesions in the control group. There was a significantly higher prevalence of dental caries in patients with UC $(\mathrm{p}=0.000)$ compared with controls with an OR for DMF-T of 2.71 (95\% CI: 1.21-7.62).

Conclusions Patients with UC had a higher prevalence of dental caries and oral lesions compared with a healthy control group. Altered dietary habits and malabsorption may probably be the main cause for this condition. Strict oral hygiene should be recommended, and the regular use of fluoride treatment for prevention of dental caries appears justified.

\section{IDDF2018-ABS-0029 ANTIOXIDANT BIOMARKERS IN ACUTE PANCREATITIS}

Le Thi Thu Hien*. Department of Internal Medicine, Thai Nguyen University of Medicine and Pharmacy, Vietnam

\subsection{6/gutjnl-2018-IDDFabstracts.74}

Background There has been increasing awareness of the role played by oxidative stress in many inflammatory illnesses. In several models, it has been demonstrated that oxygen-derived 
free radicals are generated during acute pancreatitis (AP). It has been suggested that free radical generation is an important factor in the pathogenesis of AP. There is a convincing body of evidence that antioxidant blood levels diminish during severe AP.

Aims The study aims to estimate the changes in the serum levels of Total Antioxidant Status(TAS), erythrocyte enzymatic antioxidants: Superoxide Dismutase (SOD) and Glutathion peroxidase (GPx) in AP disease and assess whether TAS is a useful biomarker to prognosis AP patients.

Methods This study was conducted in General Medicine Department of Thai Nguyen Nation Hospital, in Vietnam. TAS, SOD and GPx were measured in 83 cases of AP and 62 healthy volunteers which were regarded as the control group. Using ELISA kit supplied by Wkea Med Supplies Corp, China.The severity of pancreatitis was scored using Computerised Tomography (CT) severity index, modified severity index and revised Atlanta classification and classified into mild, severe categories.

Results A significant decrease $(\mathrm{p}<0.001)$ in the level of TAS was observed in the study group as compared to that in the control group. A significant decrease $(p<0.05)$ in the level of SOD was observed in the study group as compared to that in the control group. A significant decrease $(p<0.05)$ in the level of GPx was observed in the study group as compared to that in the control group. TAS levels of severe AP patients were statistically lower than mild AP $(10.14 \mathrm{U} / \mathrm{ml}$ vs $14.06 \mathrm{U} / \mathrm{ml}$, $\mathrm{p}<0.01)$.

Conclusions Reduced antioxidants suggest that oxidative stress plays a vital role in the pathogenesis of AP. The results of this study demonstrated changes in antioxidant biomarker associated with the prognosis AP patients. TAS could be used as a antioxidant biomarker of disease severity.

\section{IDDF2018-ABS-0032 CORRELATION OF BMI AND COLONOIC POLYPS AND ITS HISTOPATHOLOGY: A RETROSPECTIVE REVIEW}

Patricia Te*. Chinese General Hospital and Medical Center, Philippines

\subsection{6/gutjnl-2018-IDDFabstracts.75}

Background An estimated 5.7 million Filipinos are affected by obesity. Obesity has been linked to various cancers. Colon cancer is among the top five of cancers in the Philippines. Colonic Polyps have the tendency to develop into carcinoma. This study aims to find a correlation between the BMI with the risk of developing colonic polyps and pave the way for more effective screening tests for colon cancer.

Methods Patients who underwent colonoscopy for various purposes from January to October 2017 were retrospectively analysed. Age, gender, BMI, symptoms, type and location of the colonic polyp, and histopathology were investigated. SPSS v20 was used for statistical analysis.

Results There were 1204 patients who underwent successful total colonoscopy. There were 383 (31.811\%) who had colonic polyps; with 162 (42.3\%) females and $221(57.7 \%)$ males. Age, gender, and BMI were significantly correlated with the risk of developing colonic polyps $(\mathrm{p}<0.018)$. Colonic polyps present at the 6 th decade of life. They are mostly incidental findings on routine checkup, with change in bowel habit the second most common presenting symptom $(p=0.000)$. Most colonic polyps are left sided, particularly at the sigmoid colon $(p=0.000)$. The most common histologic type are hyperplastic and adenoma $(\mathrm{p}=0.000)$.

\begin{tabular}{llll}
\multicolumn{4}{c}{ Abstract IDDF2018ABS0032 Table 1} \\
\hline & With Polyp 31.811\% N=383 & Control 68.189\% N=821 & p value \\
\hline Age & $60.56 \pm 12.604$ & $54.82 \pm 15.461$ & \\
$\quad 50$ yo & $18.02 \%(n=69)$ & $36.22 \%(n=297)$ & 0.000 \\
$>50$ yo & $81.98 \%(n=314)$ & $63.78 \%(n=354)$ & \\
Gender & & & \\
female & $42.3 \%(n=162)$ & $56.88 \%(n=467)$ & 0.000 \\
$\quad$ male & $57.7 \%(n=221)$ & $43.12 \%(n=354)$ & \\
Symptom & & & \\
screening & $40.78 \%(n=146)$ & $26 \%(n=197)$ & \\
hematochezia & $17.88 \%(n=64)$ & $23.06 \%(n=175)$ & 0.000 \\
$\quad$ bowel habit & $18.72 \%(n=67)$ & $18.2 \%(n=138)$ & \\
BMI & $19.183 \pm 11.65$ & $18.804 \pm 14.56$ & \\
$\quad$ underweight & $2.42 \%(n=7)$ & $6.65 \%(n=41)$ & \\
normal & $37.37 \%(n=108)$ & $42.79 \%(n=264)$ & 0.018 \\
$\quad$ overweight & $28.37 \%(n=82)$ & $25.45 \%(n=157)$ & \\
$\quad$ obese & $31.83 \%(n=92)$ & $25.12 \%(n=155)$ & \\
\hline
\end{tabular}

Conclusions Colonic polyps are associated with increasing age, BMI and male gender. These factors should be accounted for in screening patients for colorectal cancer.

$\begin{array}{ll}\text { IDDF2018-ABS-0034 } & \text { HIGH AGE-SPECIFIC PREVALENCE OF } \\ & \text { INFLAMMATORY BOWEL DISEASE } \\ & \text { AMONGST THE ELDERLY IN THE CITY OF } \\ & \text { CANADA BAY AREA, SYDNEY: A } \\ & \text { METROPOLITAN, POPULATION-BASED } \\ & \text { STUDY }\end{array}$

${ }^{1}$ Jeffrey Liu*, ${ }^{1}$ Viraj Kariyawasam, ${ }^{2}$ Thomas Borody, ${ }^{1}$ Peter Katelaris, ${ }^{1}$ Webber Chan, ${ }^{1}$ Cowlishaw James, ${ }^{1}$ Charles McDonald, ${ }^{1}$ Grace Chapman, ${ }^{3}$ Crispin Corte, ${ }^{3}$ Warwick Selby, ${ }^{4}$ Daniel Lemberg, ${ }^{5}$ Cheng Lee, ${ }^{1}$ Anil Keshava, ${ }^{6}$ John Napoli, ${ }^{2}$ Robert Clancy, ${ }^{1}$ Rupert Leong. ${ }^{1}$ Concord Repatriation General Hospital, Sydney, Australia; ${ }^{2}$ Centre for Digestive Diseases, Sydney, Australia; ${ }^{3}$ Royal Prince Alfred Hospital, Sydney, Australia; ${ }^{4}$ Sydney Children's Hospital, Sydney, Australia; ${ }^{5}$ Westmead Children's Hospital, Sydney, Australia; ${ }^{6}$ Ryde Hospital, Sydney, Australia

\subsection{6/gutjnl-2018-IDDFabstracts.76}

Background Knowledge of Inflammatory Bowel Disease (IBD) prevalence allows health care administrators to understand disease burden and appropriately plan for research and medical care. Young IBD subjects often migrate from rural to urban areas for education and work opportunities, necessitating metropolitan prevalence studies to reduce under-representation. Also, the impact of urbanisation on IBD prevalence requires further exploration. Unlike IBD incidence, where young agegroups dominate, we hypothesised that the elderly age-groups would have the highest IBD prevalence given mortality rates being equivalent to the general population. We aimed to determine the first IBD prevalence rates for New South Wales, Australia.

Methods This was an observational, population-based epidemiological study which captured disease information of people living with IBD within the metropolitan City of Canada Bay Local Government Area on the 1st of January 2016. The diagnosis was according to the Copenhagen Criteria. Agestandardisation was according to the WHO Standard Population. 